\title{
Effect of Active Galactic Nuclei (AGN) on the Photometric and Morphologic Properties of NGC 4414 and NGC 4369 Spiral Galaxies
}

\author{
Huda Hardan * and Abdullah K. Ahmed ** \\ Department of Astronomy and Space, College of Science, University of Baghdad, Baghdad-Iraq. \\ * Corresponding Author: hudahardan1994@gmail.com, \\ ** Corresponding Author: abdullahahmed1977@gmail.com
}

\section{Abstract}

The purpose of this work is to clarify the effect of the Active Galactic Nucleus (AGN) on the properties of the galaxy. A photometric study of two galaxies by surface optical measurements techniques and by using'griz filters' was performed. The scientific material that used in this work was obtained from "SLOAN DIGITAL SKY SURVEY" (DR7), a fuzzy color, contour maps, photometric parameters, and color indices were studied by using surface photometric technique. The work was done by Ellipse task in IRAF (Image Reduction and Analysis Facility) software from the National Optical Astronomy Observatory (NOAO).

[DOI: 10.22401/ANJS.22.1.09]

Keywords: Active Galactic Nuclei, Spiral Galaxy, Surface Photometry, and individuals: NGC4414, NGC4369.

\section{Introduction}

Surface photometry is used to study the extended objects, such as galaxies, as opposed to pointing sources, such as stars. Surface photometry of galaxies is a method to define quantitatively the light distribution of the galaxies, as recorded in 2- dimensional images [1]. From an image of a point source, only its total magnitude can be derived but to the galaxy it is possible to determine the number of quantities, such as how the intensity and ellipticity vary with radius [2]. In this study, two active galaxies have been chosen, which is as follows:

NGC 4414 Classified (Sab) bright nuclear point source embedded in a small elliptical bulge. The inner disk has a different position Angle than the bulge. Spiral features appear in the inner disk are brightest here. The disk is very knotty, with evidence for many bits of arms. the pattern Spiral is flocculent [3]. NGC 4414 is a multi-armed flocculent spiral of medium inclination in the Virgo cluster. In the Hubble Space Telescope (HST) project, the Cepheid distance of NGC 4414 was determined to $19.1 \mathrm{Mpc}$ [4], NGC 4414 has flocculent spiral arms and active star-forming regions spread over the disk ,as shown in Fig.(1)[5].

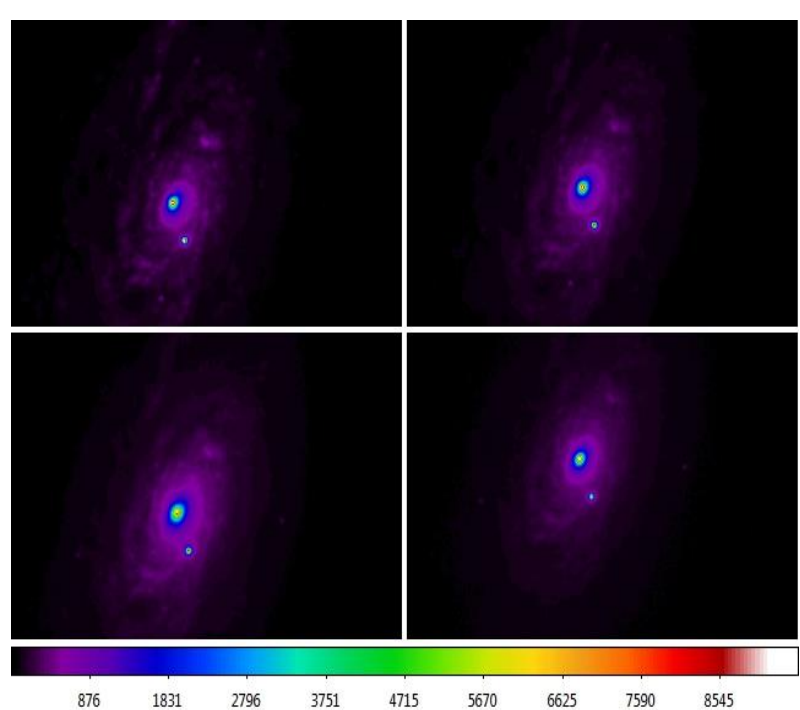

Fig.(1): Fuzzy color images of NGC 4414 active galaxy with griz-Filters, from left to right. North is up and East is at left.

NGC 4369 or Mkn 439 is a nearby early-type galaxy, it has been classified as a starburst by Balzano (1983) based on the equivalent width of $\mathrm{H} \alpha$ and also belongs to the IRAS Bright Galaxy Sample. Rudnick \& Rix (1998) report an azimuthal asymmetry in the stellar mass distribution of Mkn 439 based on the R band surface brightness. The galaxy image was nearly circular and appeared featureless in long exposure images. The outer isophotes were smooth and nearly circular in $\mathrm{B}$ and $\mathrm{R}$ bands. However, the isophotal contours show highly complex features in the inner parts. Moreover, the strength of these features is waveleng, as shown in Fig.(2). 

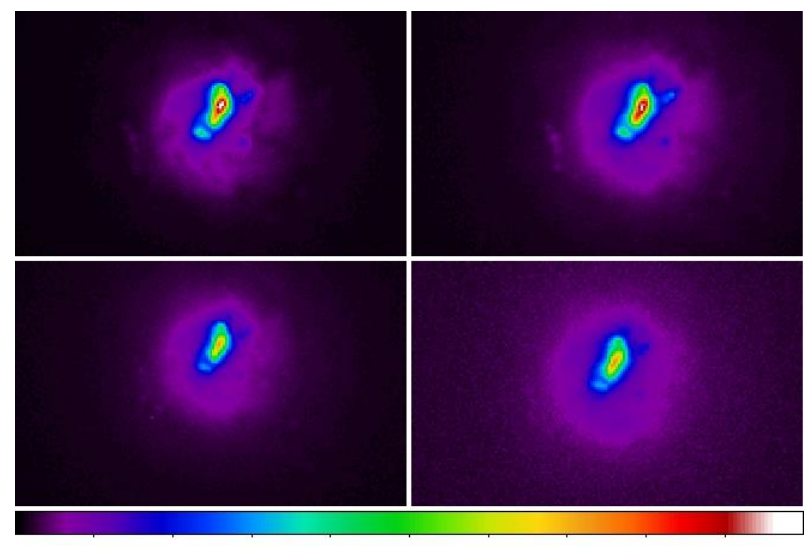

Fig. (2): Fuzzy color images of NGC 4369

active galaxy with griz-Filters, from left to right. North is up and East is at left.

\section{Materials and Methods}

The data for the two galaxies is obtained from the seventh Sloan Digital Sky Survey (SDSS) Data Release (DR7), which is reach now to (DR14)[7]. The bias and flat field were corrected for all images by SDSS pipeline. The reduction of the data was carried out using the procedures in the ${ }^{1}$ IRAF image-reduction Package. The main reduction steps are as follows:

1. The sky background values is subtracted by choosing empty regions in the image frame far from objects and measures its average intensity value.

2. Masking the superimposed and nearby objects, stars or galaxies as Shown in Fig.(3).

3- The IRAF ISOPHOTE ELLIPSE task is applied to obtain the intensity and structural profiles.

4. Transform pixel units into $\operatorname{arcsec}^{2}\left(\operatorname{arcsec}^{2}\right.$ $=1.04719755 \mathrm{rad}$ ): by dividing on the scale $(1$ pixel $=0.396$ arcsec for Apache Point $2.5 \mathrm{~m}$ Observatory (APO)).

5- Dividing frames by the exposure time value given in the header (the value is the same for all filters, it equals 53.907456 seconds).

6- Correct all frames for atmospheric extinction, galactic extinction, and transform to the standard system (using the zeropoint, atmospheric extinction and airmass of the SDSS photometric system at the time of

${ }^{1}$ IRAF is distributed by the National Optical Astronomy Observatories, which is operated by the Association of Universities for Research in Astronomy, Inc., under cooperative agreement with the National Science Foundation. observation) by multiplying the counts by the factor $(f)$ where:

$f=10^{\left(z_{p}+k * \text { airmass }\right)}$

Where $z_{p}$ and $k$ are the zeropoint magnitude and the atmospheric extinction, respectively. Table (1) lists these values for the galaxy for each filter. 




Fig.(3): griz-Filters mask region, left NGC4414 and right NGC4369.

Table (1)

Correction Values: air masses, zero points, and atmospheric extinctions.

\begin{tabular}{|c|c|c|c|c|}
\hline Galaxy & Band & Air mass & Zero point & Atmospheric Extinction \\
\hline \hline \multirow{4}{*}{ NGC 4414 } & $g$ & 1.0268 & -24.401 & 0.1367 \\
\cline { 2 - 5 } & $r$ & 1.0235 & -24.039 & 0.0908 \\
\cline { 2 - 5 } & $i$ & 1.0243 & -23.61 & 0.0368 \\
\cline { 2 - 5 } NGC 4369 & $z$ & 1.026 & -21.965 & 0.0345 \\
\cline { 2 - 5 } & $g$ & 1.0268 & -24.401 & 0.1367 \\
\cline { 2 - 5 } & $r$ & 1.0235 & -24.039 & 0.0908 \\
\cline { 2 - 5 } & $z$ & 1.0243 & -23.616 & 0.036 \\
\hline
\end{tabular}

7- Convert to magnitude units using the formula [8]:

$\boldsymbol{m}=-2.5 \log (I)$

Where $\boldsymbol{m}$ is the apparent magnitude and $I$ is the intensity. Throughout this work .Hubble constant was assumed to: $\mathrm{HO}=74 \pm 4 \mathrm{kms}^{-1}$ $\mathrm{Mpc}^{-1}$ for NGC 4414 and $75 \mathrm{kms}^{-1} \mathrm{Mpc}^{-1}$ for NGC 4369. Given the adopted distance to NGC 4414 from the Tully et al. (2013), which is about 17.9 $\mathrm{Mpc}$ [9], and 21.6 Mpc with Tully-Fisher (1988) from NASA/IPAC EXTRAGALACTIC DATABASE (NED) to NGC 4369. The image scale for NGC 4414 was $86.8 \mathrm{pc} / \mathrm{arcsec}$ and $104.7 \mathrm{pc} / \mathrm{arcsec}$ for NGC 4369[9].

\section{Results and Discussion}

Morphological Analysis

The griz-images of NGC 4414 spiral galaxy were shown in Fig.(1). The galaxy is spiral system with an (AGN) to about 2.1 arcsec embedded in an elliptical bulge with 16.2 arcsec. Spiral features appear in the inner disk and are brightest here. The disk is a very knotty, with evidence for many bits of arms. The end of disk 133.6 arcsec. The grizisophotal contour maps of NGC 4414 are shown in Fig.(4). The surface brightness levels are listed in Table (2).

Fig.(5) shows the griz-images of the NGC 4369 galaxy. It has a nucleus (AGN) component that is 1.85 arcsec, very weak spiral structure and a boxy bulge component with 17.4 arcsec diameter with fainter envelopes extended to the end of the galaxy which is approximately 73.08 arcsec. 
Huda Hardan

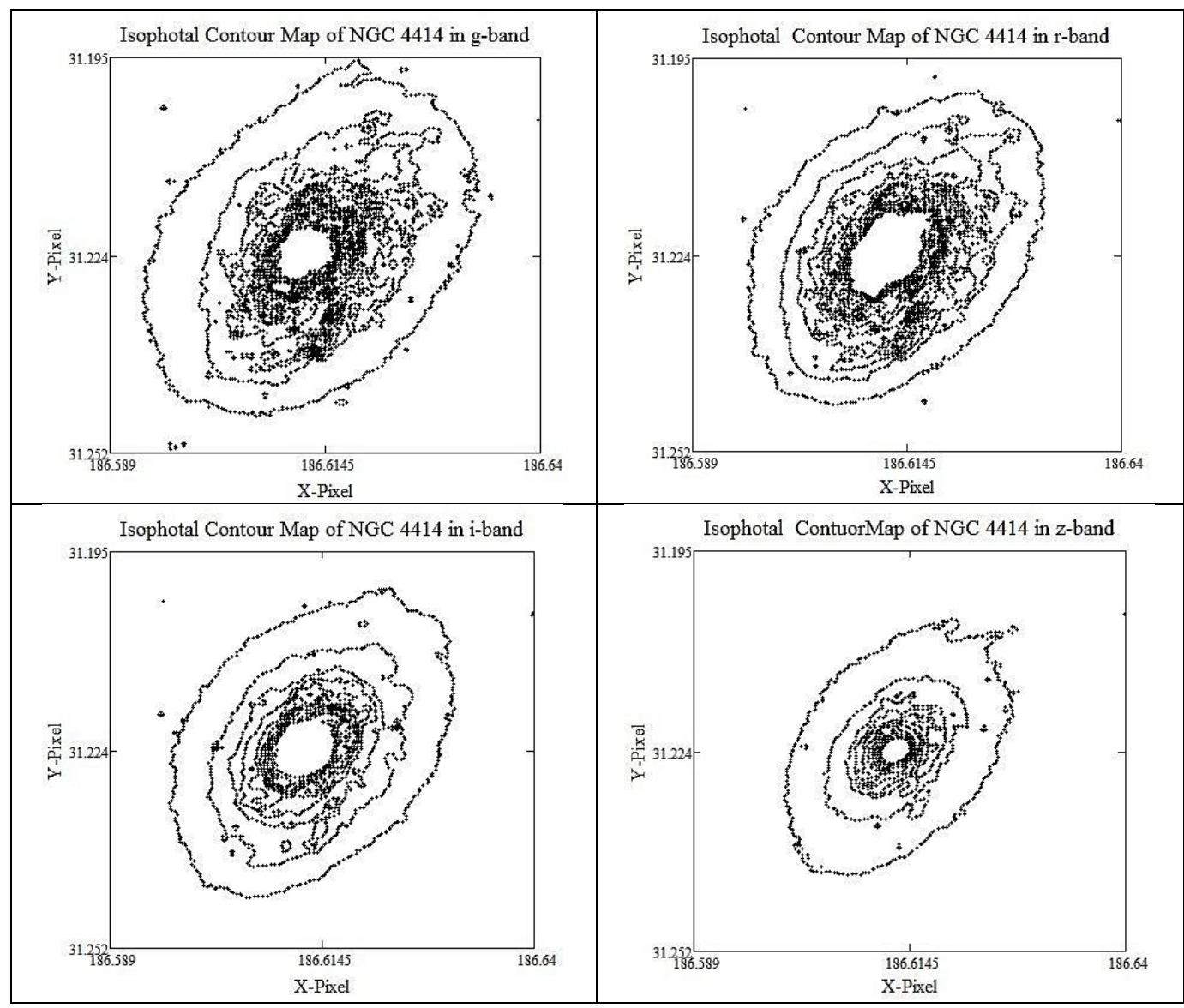

Fig.(4): Isophotal Contour Maps of NGC 4414 Galaxy in gri and z-bands, North is up and East is at left.

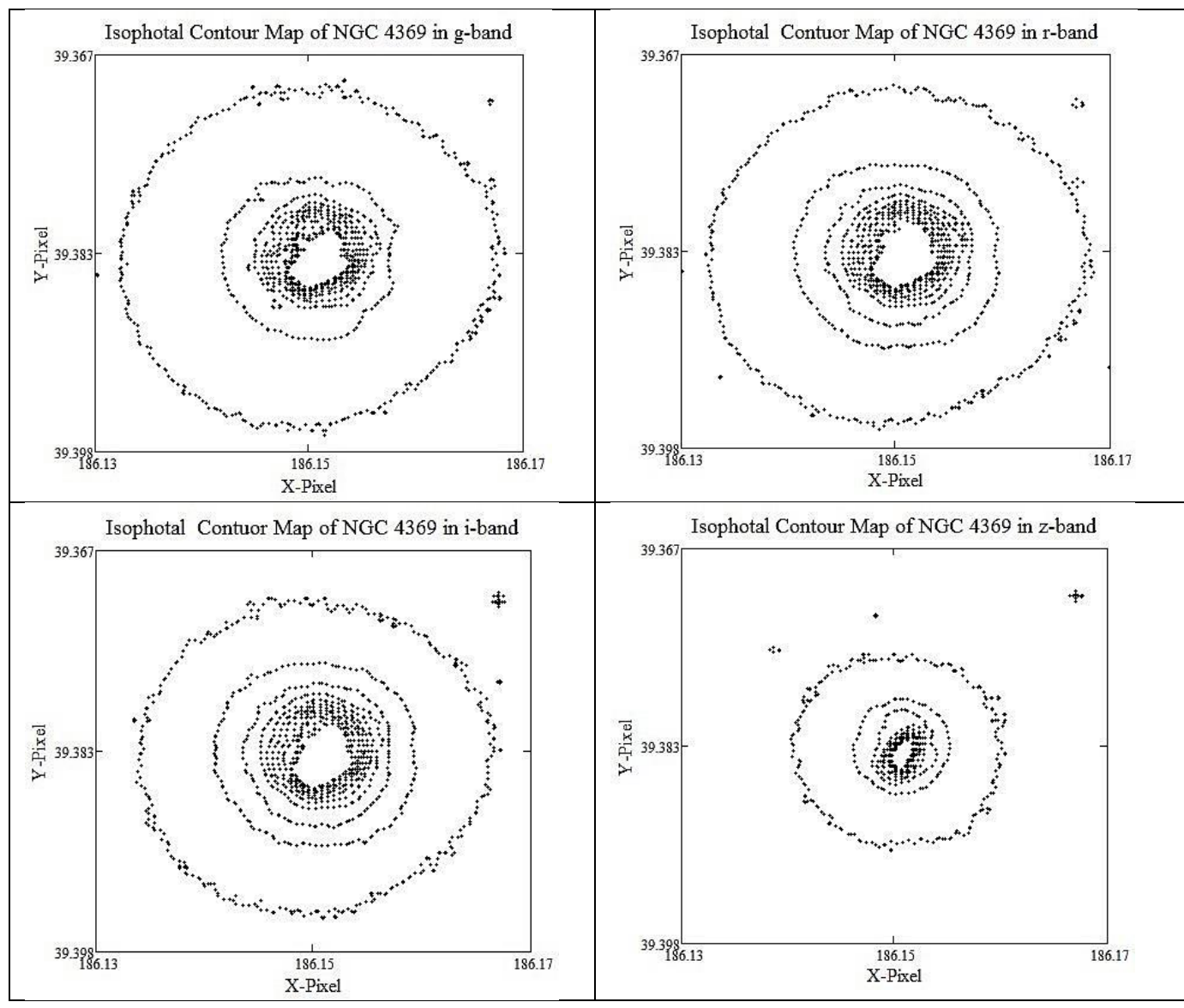

Fig.(5): Contour Maps of NGC 4369 Galaxy in gri and z-bands, north is up and East is at left. 
Table (2)

Outer isophotal level and steps of the contours of NGC 4414 and NGC 4369 galaxies.

\begin{tabular}{|c|c|c|c|c|}
\hline Galaxy & Band & -Outer isophot level (mag) & Outer isophot level (mag/arcsec $\left.{ }^{2}\right)$ & Steps \\
\hline \multirow{4}{*}{$\begin{array}{c}\text { NGC } \\
4414\end{array}$} & $\bar{g}$ & 25.129 & 23.117 & 1.269 \\
\hline & $r$ & 25.084 & 23.072 & 1.723 \\
\hline & 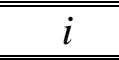 & 23.641 & 21.629 & 0.837 \\
\hline & $z$ & 22.801 & 20.79 & 1.269 \\
\hline \multirow{4}{*}{$\begin{array}{c}\text { NGC } \\
4369\end{array}$} & 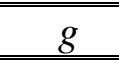 & 25.657 & 23.645 & 1.49 \\
\hline & $r$ & 25.417 & 23.406 & 1.549 \\
\hline & 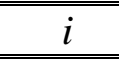 & 24.461 & 22.45 & 1.117 \\
\hline & $z$ & 23.314 & 21.303 & 1.49 \\
\hline
\end{tabular}

\section{Position Angle, Ellipticity, and B4 Profiles}

The position angle (PA), ellipticity $(\varepsilon)$ and 4th harmonic deviations from ellipse (B4) of the two galaxies isophotes were obtained as a function of the radius $(\boldsymbol{r})$, the distance from the center of the galaxy using the ellipse task of the STSDAS library in IRAF image-reduction system. The dependences of the P.A, ellipticity and $\mathrm{B} 4$ profiles on the distance from the center of the galaxy $\boldsymbol{r}$ are shown in Figures $(6,7)$ and 8 , respectively. It is noticed that the profiles show more or less similar behavior and consistency in the different bands.

For NGC 4414 PA is Clearly fluctuate between the four filters to about $11.1 \mathrm{arcsec}$, then become constant at approximately $68.8^{\circ}$ with some fluctuation at the end of the disk at about 133 arcsec as seen in Fig.(6a). The ellipticity profiles of the galaxy increase from 0.25 in the center to about 0.511 at 25.8 arcsec, then decreases to about 0.255 at the end of disk region at $(\boldsymbol{r}=133.1$ arcsec $)$ see Fig.(7a).

The mean value of the B4 profiles -0.04 due to anomaly in g-filter, and illustrated in Figure 8a and presented in Table (3) shows that the general trend of the galaxy with rizfilters is a disky.

For NGC 4369, the PA is almost constant from the center of the galaxy to about 9.13 arcsec then fluctuate until the end of the weakened galaxy disk as seen in figure $6 b$. The ellipticity profile of NGC 4369 decreases sharply from 0.57 in the bulge to about 0.06 at 13.09 arcsec, then became steady to about 0.05 until the end of the galaxy disk at 73.7 arcsec. See Fig.(7b).
The B4 profile illustrated in Fig.(8b) and presented in Table (3) shows that the general trend of the galaxy is a boxy system with global value -0.00654 . 

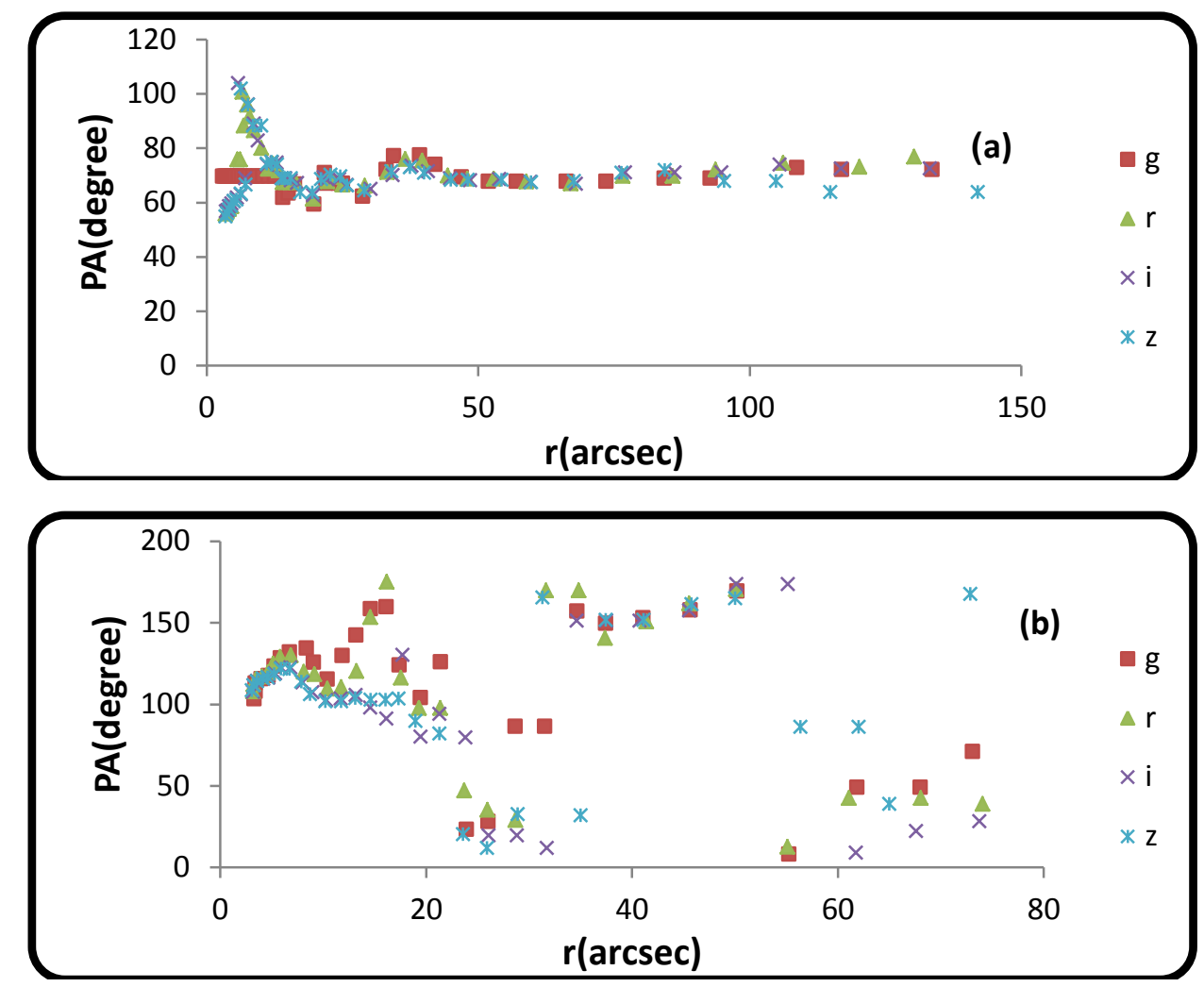

Fig.(6): Position angle profiles of a-NGC 4414, b-NGC 4369 in griz-bands.
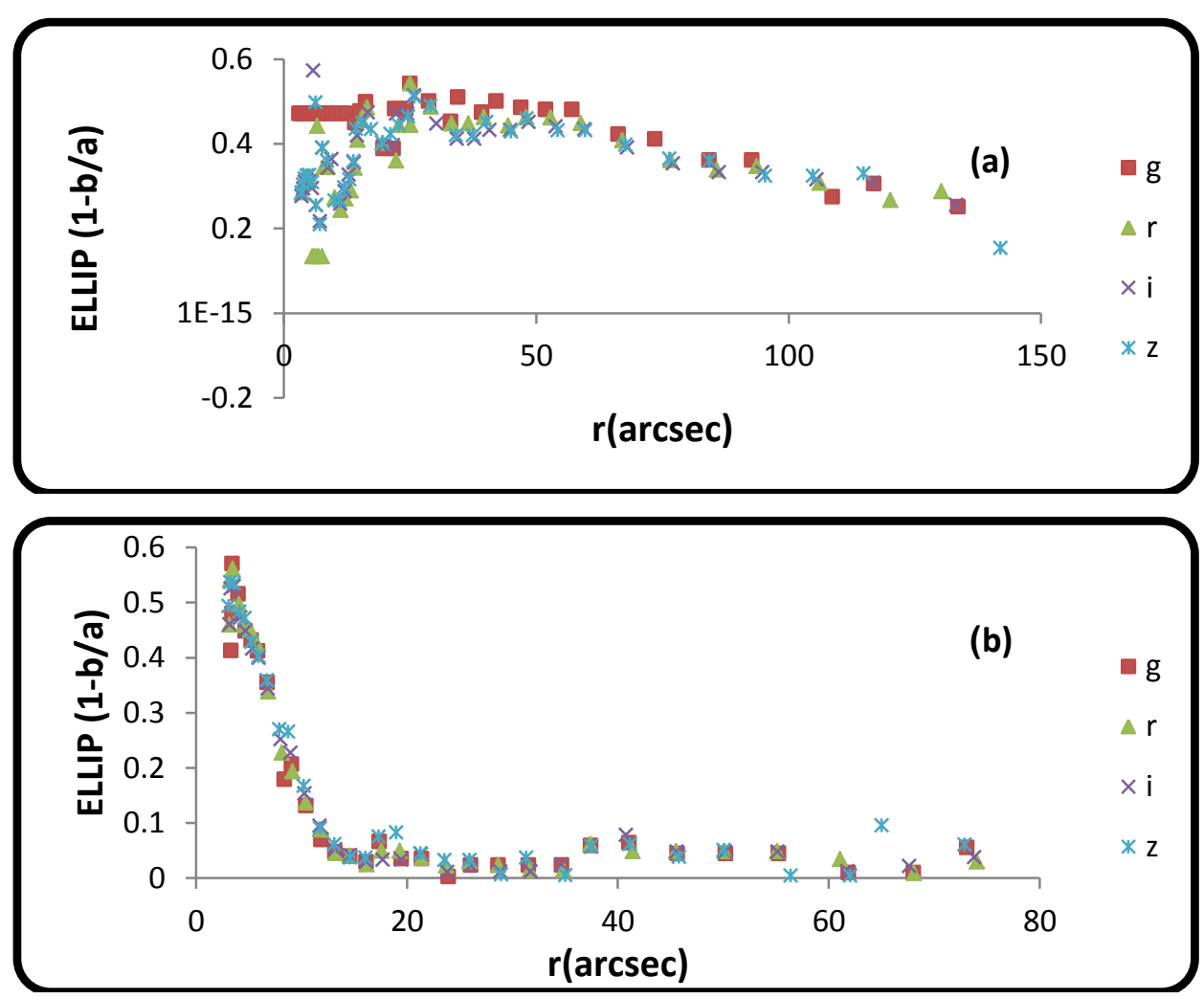

Fig.(7): Ellipticity profiles of a-NGC 4414, b- NGC 4369 in griz-bands. 

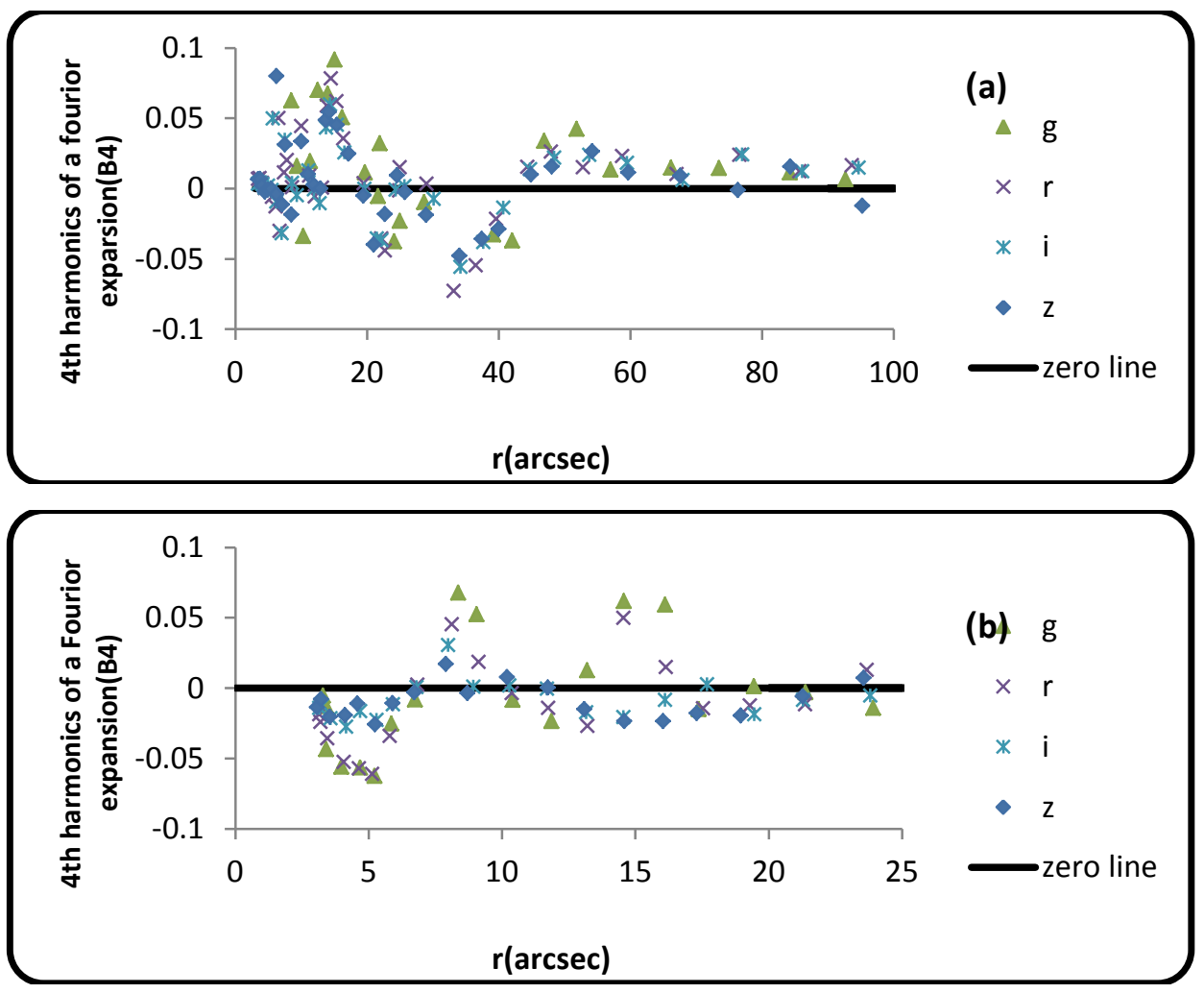

Fig.(8): B4 profiles of a- NGC 4414, b- NGC 4369 in griz-bands.

Table (3)

Isophotal position angle, Ellipticity and Inclination of NGC 4414, NGC 4369.

\begin{tabular}{|c|c|c|c|c|c|}
\hline Galaxy & Band & $\mathbf{P A}\left({ }^{\mathbf{0}}\right)$ & Ellipticity $(\varepsilon)$ & Inclination( $\left(^{0}\right)$ & $\mathrm{B} 4$ \\
\hline \multirow{5}{*}{$\begin{array}{l}\underset{Z}{J} \\
\underset{Z}{J} \\
\mathbf{Z}\end{array}$} & $g$ & $125.5 \pm 8$ & $0.40 \pm 0.109$ & $55.4 \pm 6$ & -0.18 \\
\hline & $r$ & $70.3 \pm 3$ & $0.40 \pm 0.007$ & $55.2 \pm 5.5$ & 0.00759 \\
\hline & 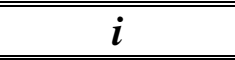 & $699.7 \pm 2.8$ & $0.39 \pm 0.06$ & $54.6 \pm 4.8$ & 0.01 \\
\hline & $z$ & $68.4 \pm 2.6$ & $0.40 \pm 0.06$ & $54.5 \pm 6.7$ & 0.00393 \\
\hline & global value & $4.834 \pm 4.1$ & $0.42 \pm 0.32$ & $54.9 \pm 5.7$ & -0.04 \\
\hline \multirow{5}{*}{$\begin{array}{l}\hat{\delta} \\
\text { †े } \\
\text { பુ } \\
\text { Z }\end{array}$} & $\bar{g}$ & $93.7 .5 \pm 64$ & $0.03 \pm 0.02$ & $15.5 \pm 5$ & -0.00382 \\
\hline & $r$ & $88.4 \pm 68$ & $0.03 \pm 0.01$ & $15.8 \pm 3$ & -0.00634 \\
\hline & 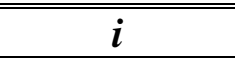 & $102 \pm 77$ & $0.04 \pm 0.02$ & $16.4 \pm 4$ & -0.01 \\
\hline & $\bar{z}$ & 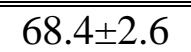 & $0.04 \pm 0.03$ & $16.08 \pm 7$ & $2-0.01002$ \\
\hline & global value & $88.1 \pm 52.9$ & $0.03 \pm 0.02$ & $15.9 \pm 4.75$ & -0.00654 \\
\hline
\end{tabular}

\section{Decomposition of Surface Brightness Profiles}

The surface brightness profiles of the NGC 4414 and NGC 4369 galaxies have been decomposed to all griz-Filters into a bulge which described by " $\boldsymbol{r}^{1 / 4}$ law" proposed by de Vaucouleurs (1948)[10] to a good approximation (Eq.3) while the disk follows an exponential brightness profile (Eq.4).

$\mu_{\text {bulge }}(r)=\mu_{e}+8.3268\left[\left(\frac{r}{r_{e}}\right)^{1 / 4}-1\right]$ $\mu_{\text {disk }}(r)=\mu_{0}+1.09\left(\frac{r}{r_{0}}\right)$

Here, $\mu_{e}$ is the surface brightness at the effective radius $r_{e}$ which is defined such that half of the brightness is emitted with in $r_{e}$. The central surface brightness and the scale-length of the disk are denoted by $\mu_{0}$ and $r_{0}$, respectively.

The results of the decomposition, fitting and the residuals values from fitting were shown in Fig.(9) and Fig.(10) of griz-Filters from upper left to right. The results were also 
summarized in Table (4), the surface brightness profiles of the two galaxies show that the outer disk of these galaxies were of type II Freeman [11].

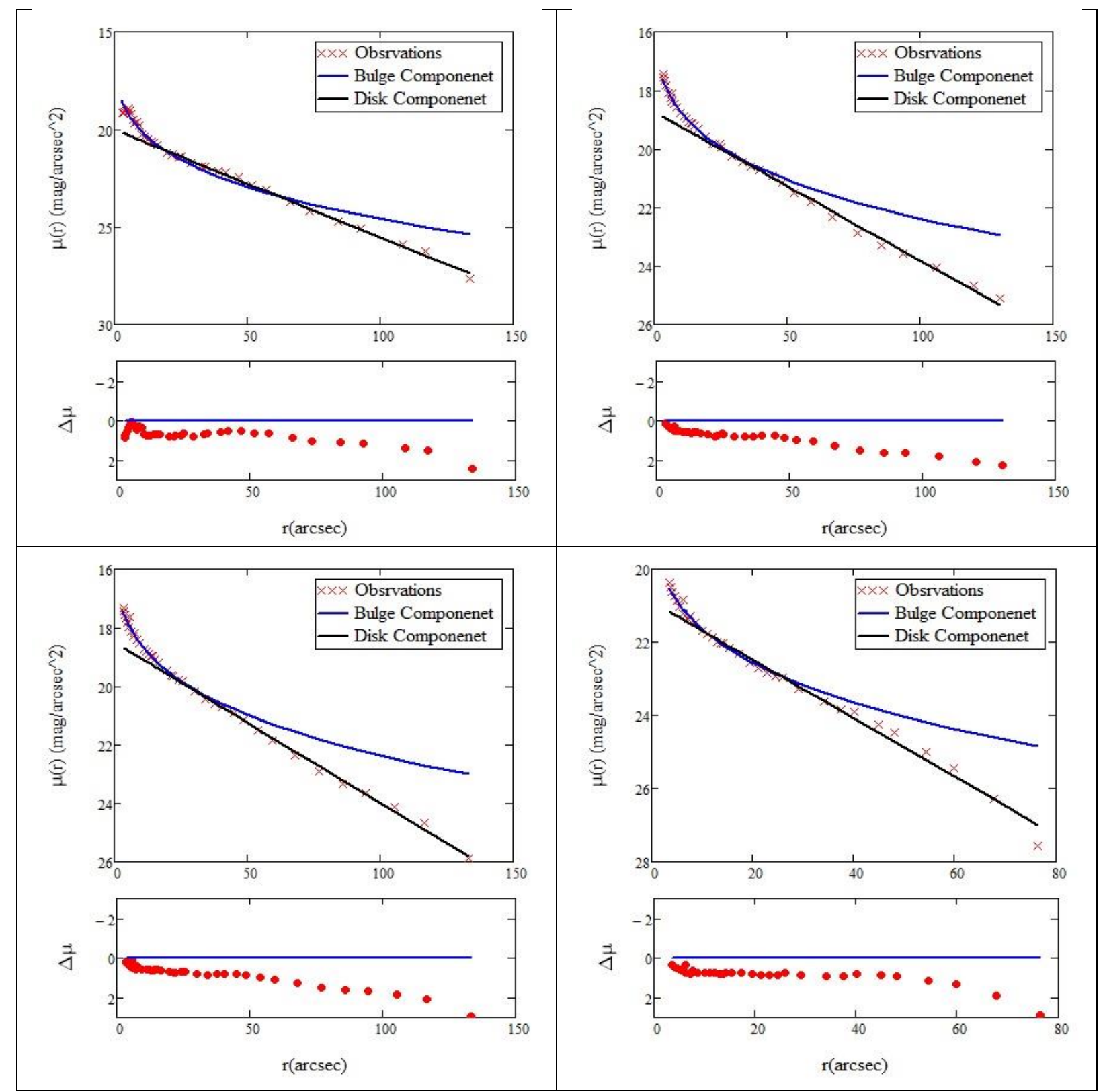

Fig.(9): Profiles decomposition of griz-Filters and the observed data for NGC 4414. griz-Filters from upper left to right. 


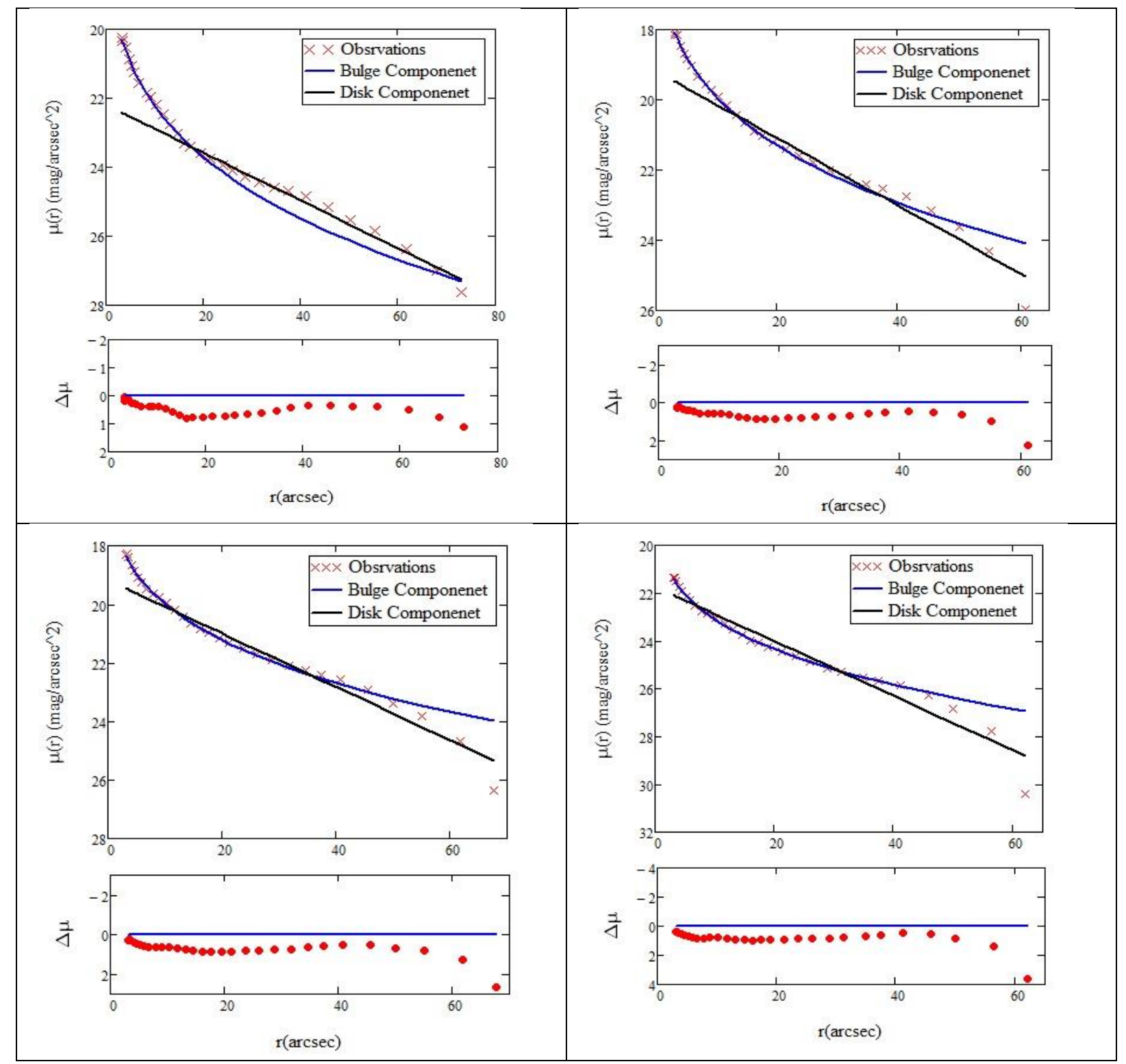

Fig.(10): Profiles decomposition of griz-Filters and the observed data for NGC 4369. griz-Filters from upper left to right.

Table (4)

Decomposition parameters of NGC 4414 and NGC 4369.

\begin{tabular}{|c|c|c|c|c|c|c|c|c|c|c|}
\hline \multicolumn{2}{|c|}{ 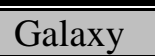 } & \multicolumn{4}{|c|}{ Bulge } & \multicolumn{3}{|c|}{ Disk } & \multirow[b]{2}{*}{ 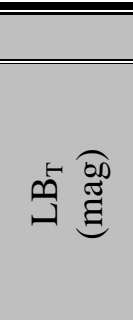 } & \multirow[b]{2}{*}{$\underset{\infty}{\stackrel{\rho}{\rho}}$} \\
\hline & $\begin{array}{l}\tilde{E} \\
\tilde{\Xi}\end{array}$ & 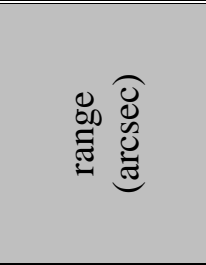 &  & $=\frac{\tilde{d}}{0}$ & 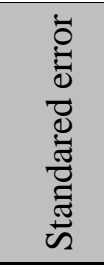 & 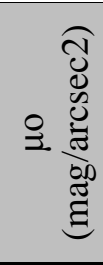 & 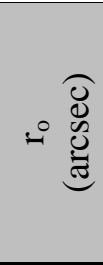 & 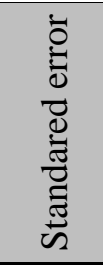 & & \\
\hline \multirow{4}{*}{$\begin{array}{l}\frac{z}{7} \\
7 \\
0 \\
\vdots \\
z\end{array}$} & $g$ & $3.632-16.24$ & 22.5 & 41.44 & 0.077 & 20.02 & 19.71 & 0.048 & 11.066 & 0.825 \\
\hline & $r$ & 4.12-16.4 & 22.33 & 97.805 & 0.027 & 18.73 & 21.36 & 0.055 & 8.995 & 1.436 \\
\hline & $\bar{i}$ & $4.147-16.6$ & 22.02 & 85.29 & 0.038 & 18.5 & 19.79 & 0.043 & 8.977 & 1.38 \\
\hline & $z$ & $4.124-17.26$ & 25.12 & 87.42 & 0.034 & 20.88 & 13.57 & 0.09 & 12.02 & 1.584 \\
\hline \multirow{4}{*}{ 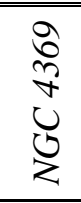 } & $g$ & $3.99-17.39$ & 22.63 & 12.34 & 0.026 & 22.19 & 15.65 & 0.065 & 13.7 & 0.782 \\
\hline & 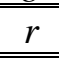 & $3.45-17.55$ & 20.79 & 17.189 & 0.021 & 19.18 & 11.32 & 0.147 & 111.14 & 0.83 \\
\hline & $\bar{i}$ & $3.57-17.69$ & 21.76 & 26.21 & 0.024 & 19.16 & 11.88 & 0.153 & 11.28 & 0.84 \\
\hline & $z$ & $3.55-17.30$ & 24.77 & 24.68 & 0.023 & 21.73 & 9.50 & 0.24 & 14.4 & 0.77 \\
\hline
\end{tabular}




\section{Color Profiles and metallicity gradients}

Wirth (1981) and Wirth and Shaw (1983) obtained colors and color gradients for a moderate sample of spirals, using aperture photometry. They find that bulges show negative color gradients, which are small in earlier-type galaxies (E, S0, Sa) and several times larger in later types ( $\mathrm{Sb}, \mathrm{Sc}$ ); They interpret these gradients in terms of metallicity gradients [14].

For NGC 4414: Profiles of $g-r, r-i$ and $i-z$ color indices, along the radius, were shown in Figure 11. This galaxy has a phenomenal color distribution where the nuclear part $(\mathrm{r} \leq 14.6$ arcsec) is blue with $g-r=0.386$ and $r-i=0.138$ but it is red for $i-z$ with value equal to -1.381 , and for the disk of this galaxy $(\mathrm{r}>14.7 \mathrm{arcsec})$ $g-r=0.319 \pm 0.063, r-i=0.075 \pm 0.056$ and $i-z=$
$-1.7 \pm 0.43$. The color indices behavior in the inner and in the outer regions of this galaxy was show anomaly for the color of normal spiral galaxies.

And for NGC 4369: Profiles of $g-r, r-i$ and $i-z$ color indices, along the radius, were shown in figure 12. This galaxy has a star-burst galactic nucleus with $(\mathrm{r}=5.7 \mathrm{arcsec})$; its effect is evident on the rest of the galaxy, where $g-r$ $=0.183 \pm 0.18, r-i=-0.0014 \pm 0.14$ and $i-z=-$ $1.52 \pm 0.16$.



Fig.(11): Color profiles of NGC 4414. 

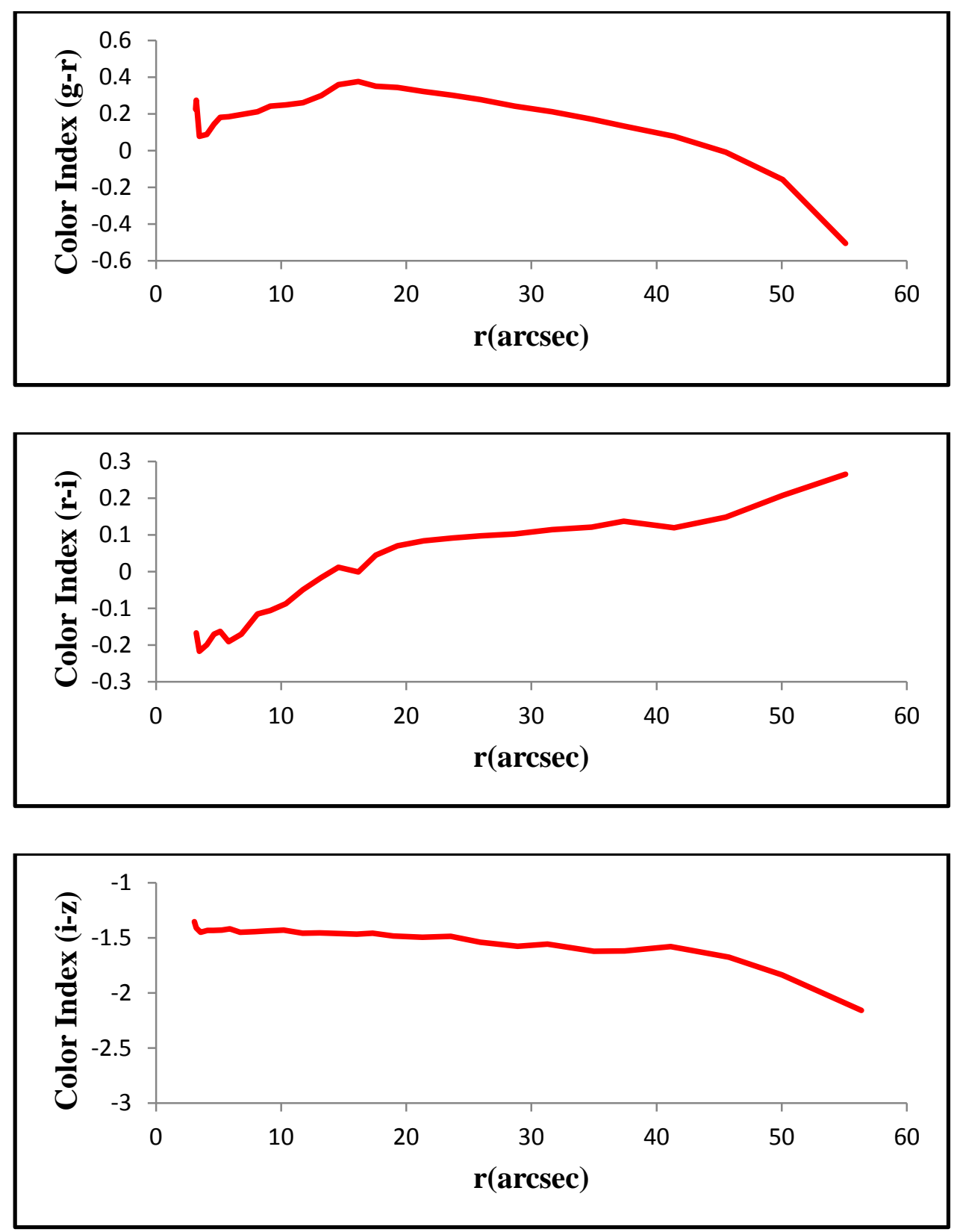

Fig.(11): Color profiles of NGC 4369.

\section{Conclusion}

1.The spiral galaxy NGC 4414 is a disky system with Bright nuclear point source embedded in a small elliptical bulge. The inner disk has a different P.A. than the bulge. Spiral features appear in the inner disk and are brightest here. The disk is very knotty, with evidence for many bits of arms. The spiral pattern is flocculent, where the barred spiral galaxy NGC4369 is a boxy system with a star-burst galactic nucleus.

2.From the results of the decomposition and fitting, were found that the type of the surface brightness profiles for the outer discs of the two galaxies is of type IIFreeman.

3.The color indices behavior in the inner and in the outer regions of these two galaxies were showed anomaly for the color of normal spiral galaxies.

\section{References}

[1] Milvang-Jensen B, and Jorgensen I, "Galaxy Surface Photometry", BalticA, 8, 535-574, 1999.

[2] Abraham R. G., van den Bergh S., Glazebrook K., Ellis R. S., Santiago B. X., Surma P. and Griffiths R. E, "The 
Morphologies of Distant Galaxies. II. Classifications from the Hubble Space Telescope Medium Deep Survey", ApJS, 107, 1, 1996 Nov.

[3] Eskridge P. B., Frogel J. A., Pogge R. W., Quillen A. C., Berlind A. A., Davies R. L. et al, "Near-Infrared and Optical Morphology of Spiral Galaxies", ApJS, 143(1), 73-111, 2002 June.

[4] Mollenhoff C., Heidt J, "Surface photometry of spiral galaxies in NIR: Structural parameters of disks and bulges", A\&A, 368: 16-37, 2001 March.

[5] Sakamoto K., Okumura S. K., Ishizuki S. and Scoville N. Z, "CO Images of the Central Regions of 20 Nearby Spiral", AJS; 1124, 403-437, 1999 Oct.

[6] Chitre A., Joshi U.C., and Ganesh S, "Star formation along a misaligned bar in the peculiar starburst galaxy Mkn 439", A\&A; 352, 112-116, 1999 December.

[7] Blanton M R, Bershady M A, Abolfathi B, Albareti F A, Prieto C A, Almeida A, et al. "Sloan Digital Sky Survey IV: Mapping the Milky Way, Nearby Galaxies, and the Distant Universe", AJ, 154(28); 35, 2017 July.

[8] Fix J D, "Astronomy Journey to the cosmic frontier, 4th ed", New York: The Mc Graw Hill Companies; 2006.

[9] Tully R. B., Courtois H. M., Dolphin A. E., Fisher J. R. et. Al, "Cosmicflows-2: The Data", AJ; 146(4), 2013 Oct22.

[10] Keel W. C, "In The Road to Galaxy Formation, Springer \& Praxis Publishing Ltd"., p.21; 2007.

[11] Freeman K C, "On the Disks of Spiral and S0 Galaxies". ApJ; 160, 811,1970 June.

[12] Balcells M. and Peletier R. F, "Colors and color gradients in bulges of galaxies", AJ, 107(1): 135-152, 1994 Jan. 